\title{
Chronological Order of Appearance of Extraintestinal Manifestations Relative to the Time of IBD Diagnosis in the Swiss Inflammatory Bowel Disease Cohort
}

\author{
Stephan R. Vavricka, MD, ${ }^{1,2}$ Gerhard Rogler, MD, PhD, ${ }^{1}$ Claudine Gantenbein, BMed, ${ }^{1}$ Muriel Spoerri, BMed, ${ }^{1}$ \\ Mareike Prinz Vavricka, MD, ${ }^{3}$ Alexander A. Navarini, MD, ${ }^{4,5}$ Lars E. French, MD, ${ }^{4}$ Ekaterina Safroneeva, PhD, \\ Nicolas Fournier, PhD, ${ }^{7}$ Alex Straumann, MD, ${ }^{8}$ Florian Froehlich, MD, ${ }^{8}$ Michael Fried, MD, ${ }^{1}$ \\ Pierre Michetti, MD, ${ }^{9,10}$ Frank Seibold, MD, ${ }^{11}$ Peter L. Lakatos, MD, ${ }^{10}$ Laurent Peyrin-Biroulet, MD, ${ }^{12}$ \\ and Alain M. Schoepfer, MD ${ }^{13}$; on behalf of the Swiss IBD Cohort Study Group
}

Background: Data evaluating the chronological order of appearance of extraintestinal manifestations (EIMs) relative to the time of inflammatory bowel disease (IBD) diagnosis is currently lacking. We aimed to assess the type, frequency, and chronological order of appearance of EIMs in patients with IBD.

Methods: Data from the Swiss Inflammatory Bowel Disease Cohort Study were analyzed.

Results: The data on 1249 patients were analyzed (49.8\% female, median age: 40 [interquartile range, 30-51 yr], 735 [58.8\%] with Crohn's disease, 483 [38.7\%] with ulcerative colitis, and 31 [2.5\%] with indeterminate colitis). A total of 366 patients presented with EIMs (29.3\%). Of those, 63.4\% presented with $1,26.5 \%$ with $2,4.9 \%$ with $3,2.5 \%$ with 4 , and $2.7 \%$ with 5 EIMs during their lifetime. Patients presented with the following diseases as first EIMs: peripheral arthritis $70.0 \%$, aphthous stomatitis $21.6 \%$, axial arthropathy/ankylosing spondylitis $16.4 \%$, uveitis $13.7 \%$, erythema nodosum $12.6 \%$, primary sclerosing cholangitis $6.6 \%$, pyoderma gangrenosum $4.9 \%$, and psoriasis $2.7 \%$. In $25.8 \%$ of cases, patients presented with their first EIM before IBD was diagnosed (median time 5 mo before IBD diagnosis: range, $0-25$ mo), and in $74.2 \%$ of cases, the first EIM manifested itself after IBD diagnosis (median: 92 mo; range, 29-183 mo).

Conclusions: In one quarter of patients with IBD, EIMs appeared before the time of IBD diagnosis. Occurrence of EIMs should prompt physicians to look for potential underlying IBD.

(Inflamm Bowel Dis 2015;21:1794-1800)

Key Words: extraintestinal manifestations, inflammatory bowel disease, arthritis, uveitis

$\mathrm{C}$ rohn's disease (CD) and ulcerative colitis (UC) represent the main entities of inflammatory bowel diseases (IBDs). During their lifetime, patients with IBD are likely to suffer from extraintestinal manifestations (EIMs), which are reported with frequencies ranging from $6 \%$ to $47 \% .^{1-8}$ The occurrence of EIM can considerably affect morbidity and mortality in patients with

Supplemental digital content is available for this article. Direct URL citations appear in the printed text and are provided in the HTML and PDF versions of this article on the journal's Web site (www.ibdjournal.org).

Received for publication November 10, 2014; Accepted March 10, 2015

From the ${ }^{1}$ Division of Gastroenterology and Hepatology, Department of Internal Medicine, University Hospital Zurich, Zurich, Switzerland; ${ }^{2}$ Department of Medicine,

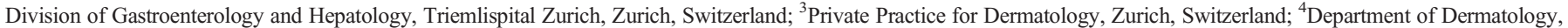

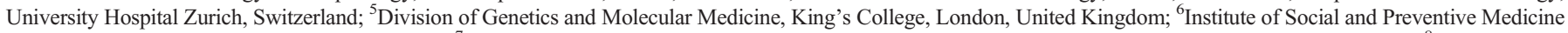

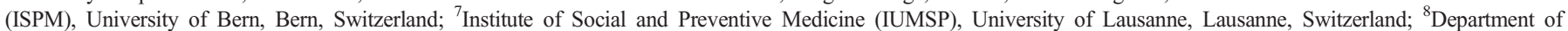

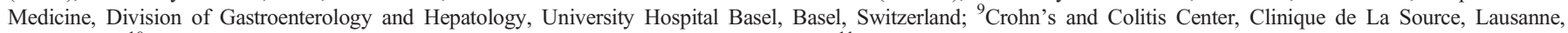

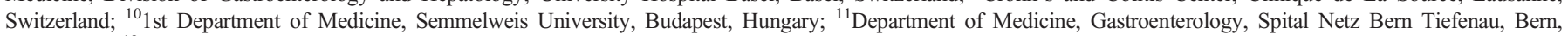

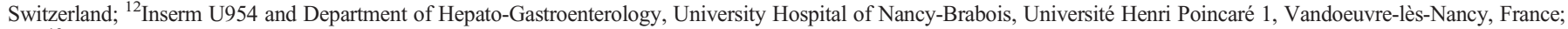
and ${ }^{13}$ Department of Medicine, Division of Gastroenterology and Hepatology, Centre Hospitalier Universitaire Vaudois (CHUV), Lausanne, Switzerland.

Supported by research Grants from the Swiss National Science Foundation (33CSC0_134274 to Swiss IBD Cohort Study group, 320000-114009/3 and 32473B_135694/1 to

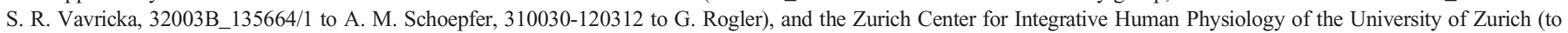
G. Rogler and S. R. Vavricka).

The authors have no conflicts of interest to disclose.

List of members of the SIBDCS study group is available in the Acknowledgments.

Reprints: Stephan R. Vavricka, MD, Division of Gastroenterology, Department of Internal Medicine, Triemli Hospital, Birmensdorferstrasse 497, CH-8063 Zurich, Switzerland (e-mail: stephan.vavricka@usz.ch).

Copyright (C) 2015 Crohn's \& Colitis Foundation of America, Inc.

DOI 10.1097/MIB.0000000000000429

Published online 27 May 2015. 
IBD. ${ }^{9,10}$ EIMs are classified into 3 major groups. The first one includes manifestations that are either associated with intestinal disease activity (peripheral arthritis, erythema nodosum, oral aphthous ulcers) or not (pyoderma gangrenosum, uveitis, spondylarthropathy, primary sclerosing cholangitis [PSC]). ${ }^{11}$ The second category of EIMs encompasses non-IBD-specific autoimmune diseases, such as thyroid disease, hemolytic anemia, vitiligo, or insulin-dependent diabetes mellitus. The third group includes IBD-related complications that are attributed to anatomical or metabolic abnormalities, such as osteopathy, nephrolithiasis, or thromboembolic events.

Most patients with CD and UC suffering from EIMs also present with extensive colitis and are likely to have a positive IBD family history. ${ }^{8,12}$ Most EIMs tend to follow the clinical course of IBD, with the exception of axial arthropathy/ankylosing spondylitis, PSC, pyoderma gangrenosum, and uveitis. ${ }^{8,13-16}$ We have recently reported that about one quarter of EIM-affected IBD patients tend to suffer from a combination of several EIMs during their disease course (up to $5 \mathrm{EIMs}$ ) ${ }^{8}$ It seems that the appearance of 1 EIM increases the susceptibility to developing other EIMs. ${ }^{8}$ The skin, joints, the biliary tract, as well as the eyes represent the organs most commonly affected by EIM. The high frequency with which these 4 organs are involved suggests a common pathogenic link; however, the underlying mechanisms are yet not completely understood. ${ }^{9}$

Despite the fact that EIMs are frequently observed in patients with IBD, data on the frequency of EIMs in large IBD populations are scarce. Furthermore, studies evaluating the chronological order of appearance of EIMs relative to the time of IBD diagnosis are lacking. As such, the proportion of patients presenting with EIMs before or after the time of IBD diagnosis has not been elucidated. The data about EIMs that precede the time of IBD diagnosis are of particular interest, since appearance of EIMs may help treating physicians to detect IBD and in doing so shorten the diagnostic delay. ${ }^{17}$

We aimed to determine the frequency of patients affected by $\operatorname{EIM}(\mathrm{s})$ before and after the time of IBD diagnosis and to assess the cumulative risk of developing one or several EIMs relative to the time of IBD diagnosis in a large national cohort of patients with IBD.

\section{MATERIALS AND METHODS}

\section{Patients}

The Swiss IBD Cohort Study (SIBDCS) has been including patients with IBD meeting the diagnostic criteria according to the established guidelines from all regions of Switzerland starting in 2006. ${ }^{18,19}$ The SIBDCS is supported by the Swiss National Science Foundation and approved by local ethics committees of the participating centers. ${ }^{20}$ For inclusion, patients need to provide written informed consent. Patients also need to have a permanent residency status in Switzerland and/or else need to be covered by a Swiss health insurance. Patients can be included into the cohort if the diagnosis of $\mathrm{CD}, \mathrm{UC}$, or indeterminate colitis (IC) was established at least 4 months before inclusion, or if they had at least 1 episode of symptom recurrence. ${ }^{20}$ At the time of inclusion, patients undergo a thorough clinical and laboratory assessment. Clinical, socioeconomic, and psychosocial data are collected. Detailed physician questionnaires are completed by patients' treating physicians, and patient-reported outcome questionnaires that, among other things, assess quality of life, the degree of social impairment and symptom severity, are completed by patients themselves. Patients were recruited in 5 University hospitals and 2 large private practices throughout Switzerland.

\section{Definitions for EIMs}

All EIMs were diagnosed by a physician. The following EIMs were analyzed: peripheral arthritis, axial arthropathy/ankylosing spondylitis, aphthous stomatitis, uveitis, erythema nodosum, pyoderma gangrenosum, psoriasis, and PSC. ${ }^{11,17,21-23}$ Rheumatologic EIMs were diagnosed by a rheumatologist. Peripheral arthritis was defined as pain, swelling, and/or redness in one or several peripheral joints. Axial arthropathy/ankylosing spondylitis was diagnosed based on a combination of typical symptoms, characteristic clinical findings, as well as x-ray examination. All patients with uveitis were diagnosed by an ophthalmologist. Erythema nodosum was diagnosed when tender bluish-red nodules (mainly on the extensor side of the limbs) were found, and pyoderma gangrenosum was diagnosed when typical chronic ulcers with undermined necrotic bluish margins (many of them beginning as pustular or nodular lesions) persisted for several weeks or longer. The skin lesions were diagnosed by dermatologists. PSC was diagnosed based on characteristic alterations of the biliary tract in endoscopic retrograde cholangiography and/or magnetic resonance cholangiography. Oral aphthous ulcers were diagnosed by the treating gastroenterologists.

\section{METHODS}

Data were entered into a Microsoft Access database (Access 2000; Microsoft Switzerland Ltd. Liab. Co., Wallisellen, Switzerland). The questionnaires were sent to the datacenter of the SIBDCS at the Institute of Social and Preventive Medicine, University of Lausanne, Switzerland. All data were validated by the data manager, and rigorous rules were applied to ensure data quality.

A standardized questionnaire, which included items on demographic characteristics, onset of symptoms, date of diagnosis, extent of disease, family history of IBD, smoking status, the results of laboratory tests, current therapy, and medical history (drugs, complications, and surgery), was completed by the treating physician. In addition, the standardized questionnaire assessed whether patients with IBD had ever experienced any kind of EIM, and whether the EIM(s) was/were present at the time of inclusion. The disease location was grouped according to the Montréal classification. For CD, L1 denotes disease in the terminal ileum, L2 denotes disease in the colon, L3 denotes ileocolonic 
disease, and L4 denotes isolated disease in the upper gastrointestinal tract. ${ }^{24}$ For UC, E1 denotes rectal disease, E2 denotes left-sided colitis, and E3 denotes extensive colitis. Since the information on the chronological order of appearance of EIM relative to the time of IBD diagnosis was not captured by the standardized questionnaires, 2 medical students (C.G. and M.S.) visited IBD centers, in which patients were included, and reviewed patient files to complete, together with the treating physician, an additional questionnaire that included items on various characteristics of EIMs. Only patients with all chronologic information regarding the appearance of EIM relative to IBD diagnosis were included. This work was performed under supervision of S.R.V. and A.M. S., who also validated all data obtained using this additional questionnaire. For the purposes of this study, data on 1249 patients with IBD, enrolled into the SIBDCS between January 2006 and March 2010, were analyzed.

\section{Statistical Analysis}

All statistical analyses were performed using the statistical package program Stata (version 12.1, StataCorp, College Station, TX). Quantitative data distribution was analyzed using Normal-QQPlots. Results of quantitative data are presented either as mean \pm $\mathrm{SD}$ and range (for Gaussian data) or median, interquartile range, and range (for non-Gaussian data). Categorical data were summarized as the percentage of the group total. Differences in categorical data distribution between groups were assessed using the chi-square test, or the Fisher's exact test in case of insufficient sample size.
Survival curves were calculated according to the KaplanMeier method to estimate probability of staying EIM free according to time from IBD diagnosis. A $P$ value $<0.05$ was considered statistically significant.

\section{RESULTS}

\section{Clinical Characteristics of the Study Population}

Of the 1249 patients with IBD included in the study, 735 patients $(58.8 \%)$ had $\mathrm{CD}, 483$ patients $(38.7 \%)$ had UC, and 31 patients $(2.5 \%)$ had IC. Six hundred twenty-two patients were female $(49.8 \%)$ with a median age at enrollment of 40 years (interquartile range, 30-51 yr). The clinical characteristics as well as disease location and current medication status of the study patients are shown in Table, Supplemental Digital Content 1, http://links.lww.com/IBD/ A874. The clinical characteristics of 366 patients (248 patients with CD, 105 patients with UC, 13 patients with IC) that presented with one or more EIMs (up to 5) are depicted in Table 1.

\section{Frequencies and Types of EIM in the Study Population}

Two hundred forty-eight patients with CD (33.7\%), 105 patients with UC (21.7\%), and 13 patients with IC (41.9\%) suffered from at least 1 EIM that occurred either before or after the time of IBD diagnosis (Table 2). The maximum number of

TABLE 1. Characteristics of Patient Affected by One or More EIMs

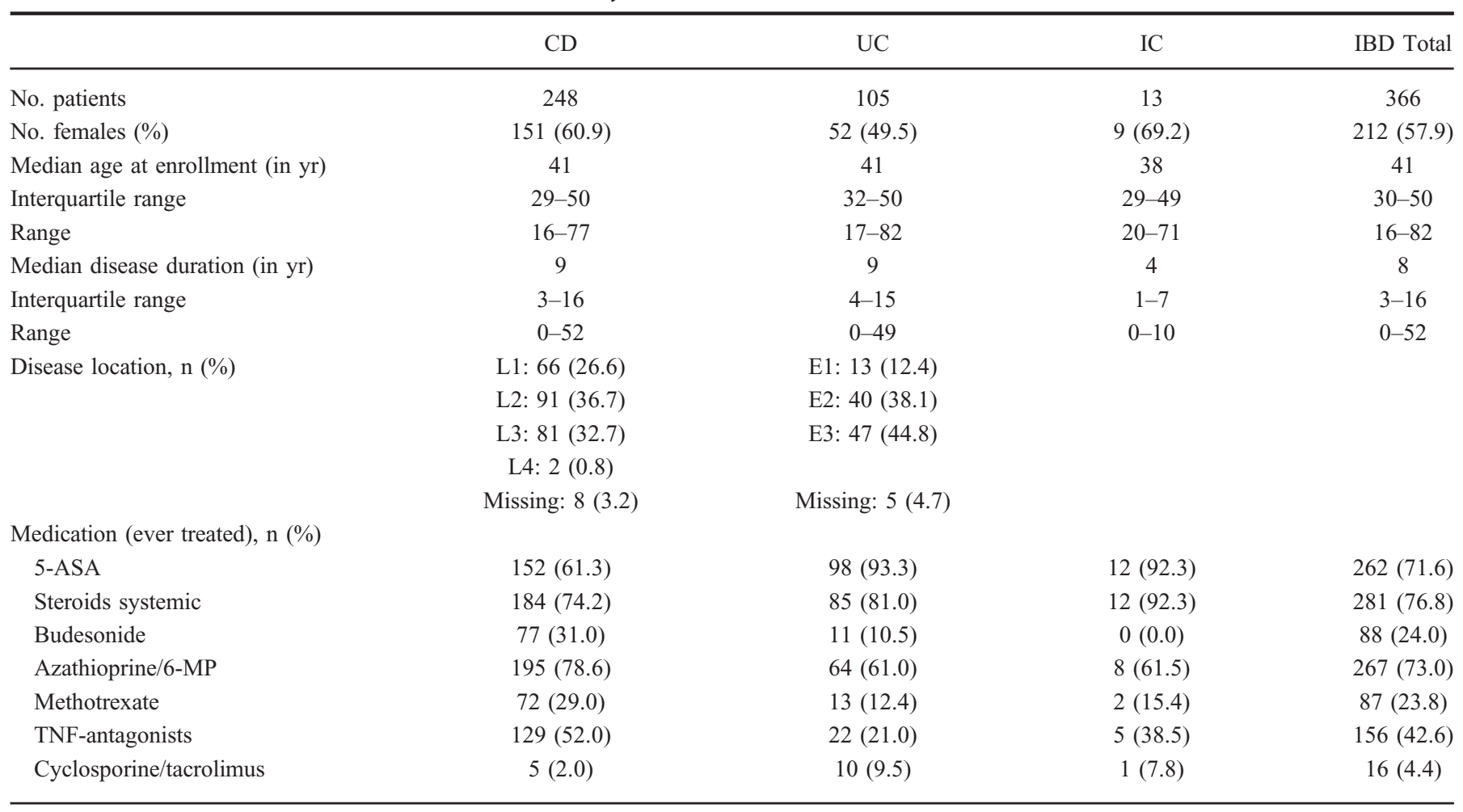

1796 | www.ibdjournal.org 
TABLE 2. Frequency and Type of EIMs in the IBD Population Examined

\begin{tabular}{|c|c|c|c|c|}
\hline & $\mathrm{CD}$ & $\mathrm{UC}$ & IC & IBD Total \\
\hline EIM frequency & 248 & 105 & 13 & 366 \\
\hline \multicolumn{5}{|l|}{ No. $\operatorname{EIM}(\mathrm{s})$ per patient, n (\%) } \\
\hline Two & $69(27.8)$ & $26(24.8)$ & $2(15.4)$ & $97(26.5)$ \\
\hline Three & $12(4.8)$ & $6(5.7)$ & $0(0.0)$ & $18(4.9)$ \\
\hline \multicolumn{5}{|l|}{ Type of EIM, n (\%) } \\
\hline Arthritis & $184(74.2)$ & $62(59.1)$ & $10(76.9)$ & $256(70.0)$ \\
\hline Aphthous stomatitis & $58(23.4)$ & $19(18.1)$ & $2(15.4)$ & $79(21.6)$ \\
\hline Axial arthropathy/ankylosing spondylitis & $45(18.2)$ & $14(13.3)$ & $1(7.7)$ & $60(16.4)$ \\
\hline Uveitis & $39(15.7)$ & $11(10.5)$ & $0(0.0)$ & $50(13.7)$ \\
\hline PSC & $5(2.0)$ & $19(18.1)$ & $0(0.0)$ & $24(6.6)$ \\
\hline
\end{tabular}

EIMs was 5 (9 patients with CD and 1 patient with UC). Of 248 patients with CD with EIM, 60.9\% had 1 EIM, 27.8\% had 2 EIM, $4.8 \%$ had 3 EIM, 2.8\% had 4 EIM, and 3.6\% had 5 EIM. During the course of their disease, 105 patients with UC who ever presented with EIMs suffered from 1 (66.7\%), 2 (24.8\%), 3 (5.7\%), 4 (1.9\%), or 5 EIMs $(1.0 \%)$. In all patients with IBD with EIMs, peripheral arthritis was the most prevalent $(70.0 \%)$, followed by aphthous stomatitis (21.6\%), axial arthropathy/ankylosing spondylitis (16.4\%), uveitis $(13.7 \%)$, erythema nodosum $(12.6 \%)$, pyoderma gangrenosum (4.9\%), PSC (6.0\%), and psoriasis $(2.7 \%)$.

\section{Distribution of Different EIMs According to Their Chronological Appearance}

The 3 most frequent EIMs, which were likely to appear as first EIM $(\mathrm{N}=232)$ were peripheral arthritis $(65 \%)$, axial arthropathy/ankylosing spondylitis (6.3\%), and aphthous stomatitis $(6.6 \%)$. If patients were diagnosed with a second EIM $(\mathrm{N}=$ 97), aphthous stomatitis (25.4\%), uveitis (20.9\%), and axial arthropathy/ankylosing spondylitis $(15.7 \%)$ were the most frequent EIM in our cohort. The most prevalent EIMs that were likely to appear as a third EIM ( $\mathrm{N}=18$ affected patients) were aphthous stomatitis (32.4\%) followed by erythema nodosum (29.7\%) and axial arthropathy/ankylosing spondylitis (13.5\%).

We then examined the time intervals in which the EIMs appeared before and/or after the time of IBD diagnosis. Figure 1 illustrates the chronological order of appearance of different EIMs in relation to the time of IBD diagnosis. Peripheral arthritis was diagnosed before the time of IBD diagnosis in $19.7 \%$ of patients. Axial arthropathy/ankylosing spondylitis was diagnosed in $39.1 \%$ of patients before IBD was diagnosed. Of note, the 25 th percentile for axial arthropathy/ankylosing spondylitis lies at minus 2 months related to IBD diagnosis. Furthermore, 5 patients experienced axial arthropathy/ankylosing spondylitis during the same month IBD was diagnosed. Aphthous stomatitis was diagnosed before IBD in $27.8 \%$ of patients, uveitis in $52.2 \%$ of patients, erythema nodosum in $14.3 \%$ of patients, pyoderma gangrenosum in $14.3 \%$ of patients, psoriasis in $50 \%$ of patients, and PSC in $23.8 \%$ of patients. Of note, musculoskeletal and dermatologic EIMs more frequently appeared after the time of IBD diagnosis rather than before $(P=0.010)$.

The Kaplan-Meier survival curves indicate the probability of staying free of EIMs in patients with CD and UC (1218 patients in total) over time (Fig. 2A). EIMs were more prevalent in

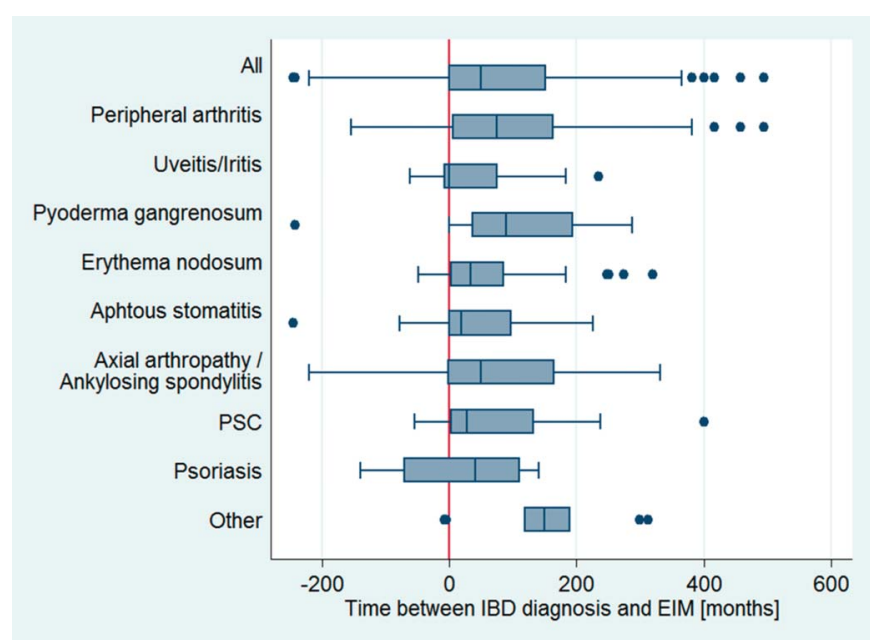

FIGURE 1. Appearance of different EIM relative to the time of IBD diagnosis. Data are presented as horizontal boxplots. The box comprises the 25 th and $75 \%$ percentile; the vertical line in the box corresponds to the median. 

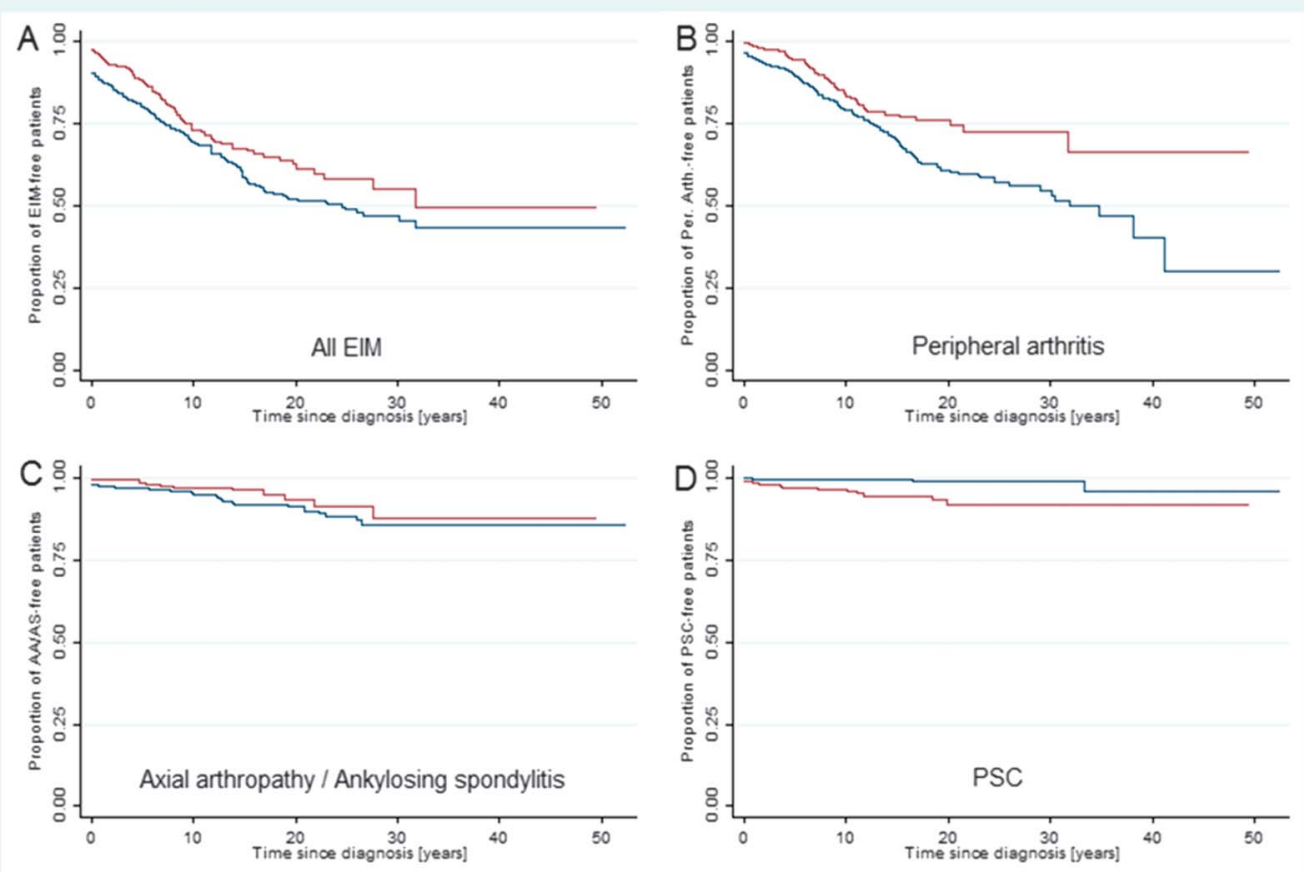

\section{Crohn's disease}

\section{Ulcerative colitis}
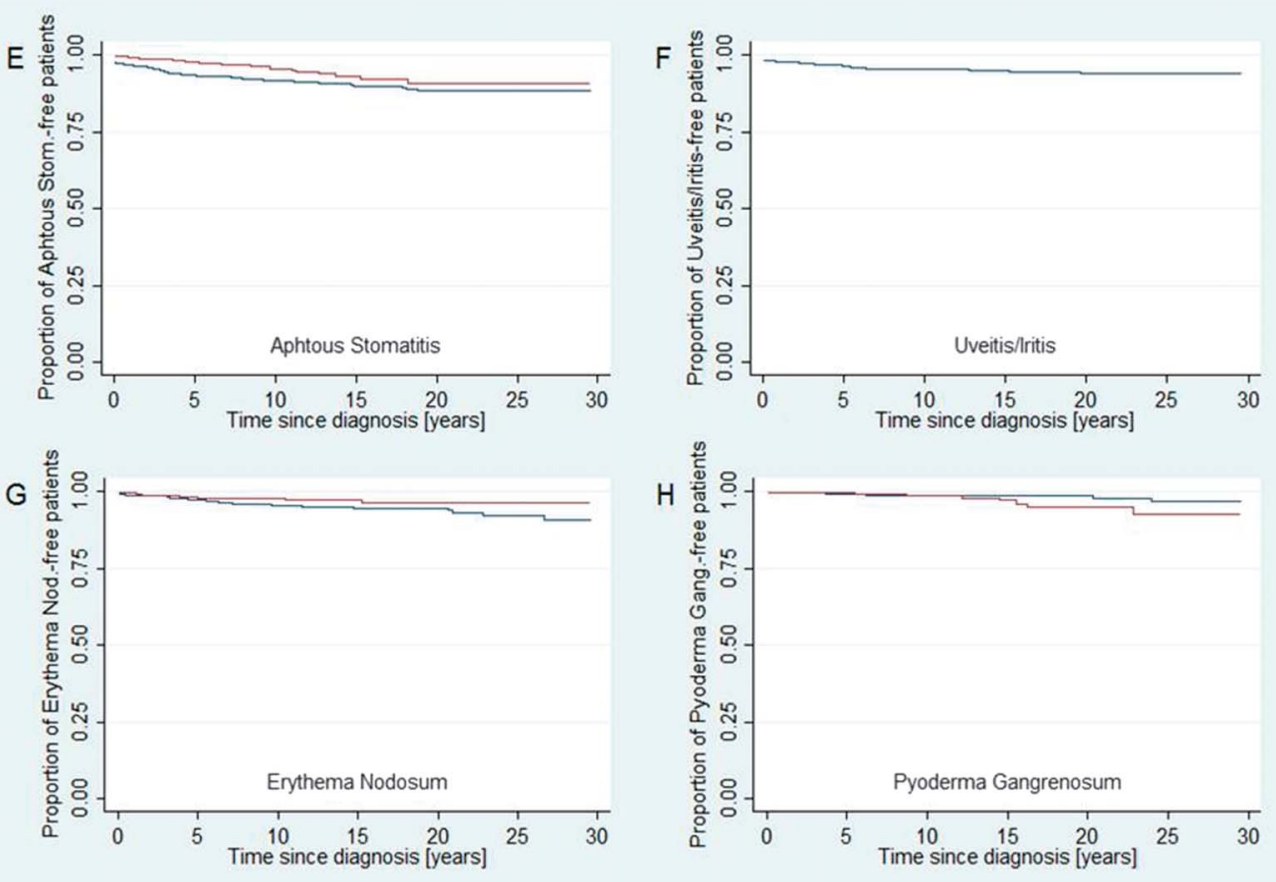

\section{Crohn's disease}

\section{Ulcerative colitis}

FIGURE 2. (A), EIM-free survival (total of 1218 patients): CD (blue curve) and UC (red curve). Kaplan-Meier estimates for all EIM (panel A), for arthritis (panel B), for axial arthropathy/ankylosing spondylitis (panel C), and for PSC (Panel D). (B), EIM-free survival (total 1218 patients with IBD): CD (blue curve) and UC (red curve). Kaplan-Meier estimates for aphthous stomatitis (panel E), for uveitis/iritis (panel F), for erythema nodosum (panel G), and for pyoderma gangrenosum (panel H). 
patients with $\mathrm{CD}$ when compared with patients with UC (Fig. 2A). Only PSC (Fig. 2D) and pyoderma gangrenosum (Fig. 2H) occurred more often in patients with UC. Peripheral arthritis (Fig. 2B) and axial arthropathy/ankylosing spondylitis (Fig. 2C) were the most prevalent EIMs in our cohort of patients with IBD. After 30 years of disease duration, $54.0 \%$ of patients with $\mathrm{CD}$ and $73.0 \%$ of patients with UC were free of peripheral arthritis.

\section{DISCUSSION}

In this large cohort study, we report on the chronologic occurrence of different EIMs relative to the time of IBD diagnosis. EIMs are common in patients with IBD, and we demonstrate that EIMs can appear in one quarter of patients before IBD is diagnosed.

In accordance with data from Veloso et al and our previous study, we found a higher frequency of EIMs in patients with CD compared with patients with UC. ${ }^{7,8}$ Veloso et al did not differentiate between the different types of EIM over time but pooled them together to evaluate the EIM prevalence during a 20-year follow-up period. Lakatos et $\mathrm{al}^{25}$ evaluated 873 Hungarian patients with IBD (254 patients with CD, average disease duration $9.2 \mathrm{yr}$; 619 patients with UC, average disease duration $11.2 \mathrm{yr}$ ) and found that a total of $21.3 \%$ had EIM (UC: $15.0 \%, \mathrm{CD}$ : $36.6 \%$ ). Although the disease duration between the Hungarian cohort and the Swiss cohort was comparable, we observed a higher EIM frequency in our cohort, particularly regarding the joint manifestations.

Importantly, we found that in one quarter of patients, the EIM appeared before IBD was diagnosed. When analyzing the specific EIM, we found that peripheral arthritis (in 19.7\%), axial arthropathy/ankylosing spondylitis (in 39.1\%), aphthous stomatitis (in 27.8\%), uveitis (in 52.2\%), erythema nodosum (in 14.3\%), pyoderma gangrenosum (in 14.3\%), psoriasis (in 50\%), and PSC (in 23.8\%) all may appear before IBD is diagnosed. This finding has important clinical implications since the awareness of this fact might decrease the diagnostic delay in patients with IBD. We recently showed that diagnostic delay is a particular concern especially in patients with $\mathrm{CD}$ where one fourth of patients require $>24$ months from symptoms onset until CD diagnosis are established. ${ }^{17}$ This finding is of particular importance since during this period bowel damage can already occur. ${ }^{26}$

The development of 1 EIM appears to increase the susceptibility of developing other EIM. ${ }^{27}$ An overlap of EIM has particularly been observed with peripheral arthritis, erythema nodosum, affections of the biliary tract, and the eyes, which support the hypothesis of a common pathogenic pathway. Supporting that concept, Bhagat et $\mathrm{al}^{28}$ reported an autoimmune reaction toward an isoform of tropomyosin (tropomyosin-related peptide), which is expressed in the eye (nonpigmented ciliary epithelium), skin (keratinocytes), joints (chondrocytes), biliary epithelium, and the gut. We found a relevant proportion of our patients with $\mathrm{CD}$ and UC suffering from several (up to 5) EIM; the prevalence of multiple EIM was again higher in patients with CD compared with patients with UC. Genetic analyses may help understanding the increased risk for multiple EIM in some patients. Patients with UC who display HLA-B8, DR3 phenotype were found to have a 10 -fold higher risk of PSC, ${ }^{29}$ and patients with UC with HLA DRB1*0103 (DR103) had a higher risk of ocular and articular manifestations. ${ }^{30}$

Our study has several strengths and also limitations. We report in a large cohort study, including a detailed additional chart review, on the chronologic appearance between EIM and IBD diagnosis. We further depict the cumulative risk of developing one or several EIM relative to the time of IBD diagnosis. Besides the large patient sample size, the prospective patient inclusion using physician questionnaires is probably less prone to underreporting of EIM prevalence compared with databases where EIM are retrospectively identified using the International Classification of Diseases codes. A potential limitation may be that the Swiss IBD cohort is not population based; some selection bias is therefore likely to be present. For instance, patients included by private practitioners are underrepresented compared with patients included by hospitals $(20 \%$ of patients with IBD are recruited by colleagues in private practice compared with $80 \%$ of patients recruited by physicians working in hospitals). Furthermore, the relative number of patients suffering from EIM is lower in this study $(29.3 \%)$ when compared with our previously published study $(38.1 \%){ }^{8}$ This is related to the fact that in this study, only patients with known chronologic appearance between EIM and IBD diagnosis were included.

In summary, we demonstrate in a large national cohort that EIMs are frequent and appear in one quarter of patients before IBD is diagnosed. Knowledge of this phenomenon may result in increased awareness for underlying IBD, thereby facilitating diagnosis and reducing diagnostic delay.

\section{ACKNOWLEDGMENTS}

Members of the SIBDCS study group: Peter Bauerfeind, Christoph Beglinger, Stefan Begré, José Bengoa, Janek Binek, Daniel Boller, Jan Borovicka, Christian Braegger, Bernard Burnand, Rafael Camara, Dominique Criblez, Philippe de Saussure, Lukas Degen, Joakim Delarive, Tobias Ehmann, Matthias Engelmann, Ali El Wafa, Christian Felley, Alain Frei, Remus Frei, Michael Fried, Michael Friedt, Florian Froehlich, Suzanne Gallot-Lavallée, Tilman Gerlach, Martin Geyer, Marc Girardin, Oliver Goetze, Horst Haack, Serge Hediger, Peter Hengstler, Klaas Heyland, Patrick Janiak, Pascal Juillerat, Vera Kessler Brondolo, Christoph Knoblauch, Gerd A. Kullak-Ublick, Michael Manz, Urs Marbet, Rémy Meier, Christa Meyenberger, Pierre Michetti, Christian Mottet, Christoph Müller, Beat Müllhaupt, Thierry Nicolet, Andreas Nydegger, Franziska Piccoli, Julia Pilz, Valérie Pittet, Ronald Rentsch, Jean-Pierre Rey, Silvia Rihs, Daniela Rogler, Gerhard Rogler, Markus Sagmeister, Bernhard Sauter, Niklaus Schaub, Susanne Schibli, Alain M. Schoepfer, Frank Seibold, Johannes Spalinger, Philippe Stadler, Michael Steuerwald, Alex Straumann, Michael Sulz, Michela Tempia-Caliera, 
Joël Thorens, John-Paul Vader, Stephan R. Vavricka, Jürg Vögtlin, Roland Von Känel, Gert Wachter, Jürg Wermuth, and Paul Wiesel.

Author contributions: study concept and design, S. R. Vavricka, M. P. Vavricka, A. A. Navarini, L. E. French, E. Safroneeva, A. M. Schoepfer; acquisition of data, S. R. Vavricka, C. Gantenbein, M. Spoerri, A. Straumann, M. Fried, P. Michetti, P. L. Lakatos, A. M. Schoepfer; analysis and interpretation of data, S. R. Vavricka, G. Rogler, C. Gantenbein, M. Spoerri, M. P. Vavricka, A. A. Navarini, L. E. French, E. Safroneeva, N. Fournier, A. Straumann, F. Froehlich, M. Fried, P. Michetti, P. L. Lakatos, L. P.-Biroulet, A. M. Schoepfer; drafting of the article, S. R. Vavricka, G. Rogler, C. Gantenbein, M. Spoerri, E. Safroneeva, N. Fournier, A. Straumann, F. Froehlich, M. Fried, P. Michetti, P. L. Lakatos, L. P.-Biroulet, A. M. Schoepfer; critical revision of the article for important intellectual content, S. R. Vavricka, G. Rogler, M. P. Vavricka, A. A. Navarini, L. E. French, E. Safroneeva, N. Fournier, A. Straumann, F. Froehlich, M. Fried, P. Michetti, P. L. Lakatos, L. P.-Biroulet, A. M. Schoepfer; statistical analysis, S. R. Vavricka, E. Safroneeva, N. Fournier, A. M. Schoepfer; obtained funding, S. R. Vavricka, G. Rogler, M. Fried, A. M. Schoepfer; technical, or material support, S. R. Vavricka, N. Fournier; study supervision, S. R. Vavricka, A. M. Schoepfer.

\section{REFERENCES}

1. Bernstein CN, Wajda A, Blanchard JF. The clustering of other chronic inflammatory diseases in inflammatory bowel disease: a population-based study. Gastroenterology. 2005;129:827-836.

2. Bernstein CN, Blanchard JF, Rawsthorne P, et al. The prevalence of extraintestinal diseases in inflammatory bowel disease: a populationbased study. Am J Gastroenterol. 2001;96:1116-1122.

3. Mendoza JL, Lana R, Taxonera C, et al. Extraintestinal manifestations in inflammatory bowel disease: differences between Crohn's disease and ulcerative colitis (in Spanish). Med Clin (Barc). 2005;125:297-300.

4. Ricart E, Panaccione R, Loftus EV Jr, et al. Autoimmune disorders and extraintestinal manifestations in first-degree familial and sporadic inflammatory bowel disease: a case-control study. Inflamm Bowel Dis. 2004;10: 207-214.

5. Rankin GB, Watts HD, Melnyk CS, et al. National Cooperative Crohn's disease study: extraintestinal manifestations and perianal complications. Gastroenterology. 1979:77:914-920.

6. Su CG, Judge TA, Lichtenstein GR. Extraintestinal manifestations of inflammatory bowel disease. Gastroenterol Clin North Am. 2002;31: $307-327$.

7. Veloso FT, Carvalho J, Magro F. Immune-related systemic manifestations of inflammatory bowel disease. A prospective study of 792 patients. J Clin Gastroenterol. 1996;23:29-34.

8. Vavricka SR, Brun L, Ballabeni PL, et al. Frequency and risk factors for extraintestinal manifestations in the Swiss Inflammatory Bowel Disease Cohort. Am J Gastroenterol. 2011;106:110-119.
9. Das KM. Relationship of extraintestinal involvements in inflammatory bowel disease: new insights into autoimmune pathogenesis. Dig Dis Sci. 1999;44:1-13.

10. Monsén U, Sorstad J, Hellers G, et al. Extracolonic diagnoses in ulcerative colitis: an epidemiological study. Am J Gastroenterol. 1990;85:711-716.

11. Danese S, Semeraro S, Papa A, et al. Extraintestinal manifestations in inflammatory bowel disease. World J Gastroenterol. 2005;11:7227-7236.

12. Kethu SR. Extraintestinal manifestations of inflammatory bowel diseases. $J$ Clin Gastroenterol. 2006;40:467-475.

13. Trost LB, McDonnell JK: Important cutaneous manifestations of inflammatory bowel disease. Postgrad Med J. 2005;81: 580-585.

14. Barrie A, Regueiro M. Biologic therapy in the management of extraintestinal manifestations of inflammatory bowel disease. Inflamm Bowel Dis. 2007;13: 1424-1429.

15. Levitt M, Ritchie J, Lennard-Jones J, et al. Pyoderma gangrenosum in inflammatory bowel disease. Br J Surg. 1991;78: 676-678.

16. Mir-Madjlessi SH, Taylor JS, Farmer RG. Clinical course and evolution of erythema nodosum and pyoderma gangrenosum in chronic ulcerative colitis: a study of 42 patients. Am J Gastroenterol. 1985;80: 615-620.

17. Vavricka SR, Spigaglia SM, Rogler G, et al. Systematic evaluation of risk factors for diagnostic delay in inflammatory bowel disease. Inflamm Bowel Dis. 2012;18:496-505.

18. Van Assche G, Dignass A, Panes J, et al. The second European evidencebased consensus on the diagnosis and management of Crohn's disease: definitions and diagnosis. J Crohns Colitis. 2010;4:7-27.

19. Stange EF, Travis SPL, Vermeire S, et al. Second European evidencebased consensus on the diagnosis and management of ulcerative colitis: definitions and diagnosis. J Crohns Colitis. 2008;2:1-23.

20. Pittet V, Juillerat $P$, Mottet $C$, et al. Cohort profile: the Swiss Inflammatory Bowel Disease Cohort Study (SIBDCS). Int J Epidemiol. 2009;38: 922-931.

21. Najarian DJ, Gottlieb AB. Connections between psoriasis and Crohn's disease. J Am Acad Dermatol. 2003;48:805-821.

22. Cohen AD, Dreiher J, Birkenfeld S. Psoriasis associated with ulcerative colitis and Crohn's disease. J Eur Acad Dermatol Venereol. 2009;23:561-565.

23. Nair R, Henseler T, Jenisch S, et al. Evidence for two psoriasis susceptibility loci (HLA and 17q) and two novel candidate regions (16q and 20p) by genome-wide scan. Hum Mol Genet. 1997;6:1349-1356.

24. Silverberg MS, Satsangi J, Ahmad T, et al. Toward an integrated clinical, molecular and serological classification of inflammatory bowel disease: report of a Working Party of the 2005 Montreal World Congress of Gastroenterology. Can J Gastroenterol. 2005;19(suppl A):5-36.

25. Lakatos L, Pandur T, David G, et al. Association of extraintestinal manifestations of inflammatory bowel disease in a province of western Hungary with disease phenotype: results of a 25 -year follow-up study. World $J$ Gastroenterol. 2003;9:2300-2307.

26. Schoepfer AM, Dehlavi A, Fournier N, et al. Diagnostic delay in Crohn's disease is associated with a complicated disease course and increased operation rate. Am J Gastroenterol. 2013;108:1744-1753.

27. Ardizzone S, Puttini PS, Cassinotti A, et al. Extraintestinal manifestations of inflammatory bowel disease. Dig Liv Dis. 2008;40(suppl 2): S253-S259.

28. Bhagat S, Das KM. A shared and unique peptide in the human colon, eye, and joint detected by a monoclonal antibody. Gastroenterology. 1994; 107:103-108.

29. Chapman RW, Varghese Z, Gaul R, et al. Association of primary sclerosing cholangitis with HLA-B8. Gut. 1983;24:38-41.

30. Roussomoustakaki M, Satsangi J, Welsh K, et al. Genetic markers may predict disease behaviour in patients with ulcerative colitis. Gastroenterology. 1997; 112:1845-1853. 